\title{
Les clichés anti-grecs chez les penseurs roumains de la seconde moitié du XIX ${ }^{e}$ siècle
}

The Romanian Thinkers' Anti-Greek Clichés in the Second Half of the 19th

Century

\section{Cécile Folschweiller}

\section{OpenEdition \\ Journals}

Édition électronique

URL : https://journals.openedition.org/ceb/4896

DOI : $10.4000 /$ ceb.4896

ISSN : 2261-4184

Éditeur

INALCO

\section{Référence électronique}

Cécile Folschweiller, « Les clichés anti-grecs chez les penseurs roumains de la seconde moitié du xIx 'e siècle », Cahiers balkaniques [En ligne], 42 | 2014, mis en ligne le 27 mai 2014, consulté le 07 juillet 2021. URL : http://journals.openedition.org/ceb/4896 ; DOI : https://doi.org/10.4000/ceb.4896

Ce document a été généré automatiquement le 7 juillet 2021.

Cahiers balkaniques est mis à disposition selon les termes de la Licence Creative Commons Attribution - Pas d'Utilisation Commerciale 4.0 International. 


\title{
Les clichés anti-grecs chez les penseurs roumains de la seconde moitié du XIX ${ }^{\mathrm{e}}$ siècle
}

\author{
The Romanian Thinkers' Anti-Greek Clichés in the Second Half of the 19th \\ Century
}

Cécile Folschweiller

1 La présence grecque dans les principautés roumaines n'a pas échappé aux phénomènes qui accompagnent généralement ce genre de situations : une communauté minoritaire et plus récemment installée que la communauté majoritaire fait le plus souvent l'objet d'un regard critique et négatif. Lorsque ce groupe prend une importance croissante dans la vie économique, sociale et politique, cette représentation peut s'objectiver en un discours, souvent virulent et xénophobe, qui ne détermine pas seulement l'image de l'autre, mais aussi la représentation de soi, de celui qui profère ces jugements, surtout dans une situation où la nation se cherche et a besoin de modèles contre lesquels se définir. La figure du Grec, dont les incarnations sont multiples dans l'histoire des Principautés (le moine orthodoxe du mont Athos, le commerçant, le précepteur lettré, le fermier, l'usurier, l'administrateur du prince nommé par le sultan ou le prince luimême durant la période phanariote) alimente pendant des décennies des discours de ce type. Une trace de son expression populaire peut être relevée dans les proverbes, par définition répertoire de clichés, et c'est une image franchement négative qui s'en dégage $^{1}$. Mais cette profonde et violente attitude anti-grecque en Roumanie dépasse largement celle des "mentalités populaires ", des images de l'étranger qui résultent des relations et confrontations les plus prosaïques et les moins réfléchies qui animent ces sociétés mêlées de la Valachie urbaine. Lucian Boia montre bien que cette mythologie négative de l'Autre en Roumanie relève aussi et surtout d'une construction intellectuelle et idéologique de la part de l'élite, construction fortement liée à l'élaboration de l'idée nationale et au contexte dans lequel elle s'est faite ${ }^{2}$.

2 Les Grecs ne sont pas les seuls à avoir servi de repère négatif. De nombreux travaux ont été consacrés aux Juifs et à l'antisémitisme roumain qui court sur les deux derniers 
siècles. La figure du Grec s'est effacée plus tôt (et la communauté grecque ne fut pas l'objet, heureusement, des mêmes persécutions), la haine dont elle a fait l'objet ayant représenté une phase propre au XIX ${ }^{\mathrm{e}}$ siècle (du moins pour sa forme idéologiquement argumentée) qui va progressivement s'éteindre au tournant du siècle avec la reconsidération du rôle des Grecs opérée par Nicolae Iorga grâce au thème de Byzance ${ }^{3}$. Reste que les textes de la seconde moitié du XIX ${ }^{e}$ siècle frappent par la récurrence et la violence de cette autre image de l'Autre contre qui on se définit et on s'affirme. Eminescu, dont on connaît l'antisémitisme virulent, peut écrire, en parlant des Grecs, en 1881, que « cette plèbe roumanisée depuis Tudor [Vladimirescu] joue en Valachie le même rôle que les Juifs en Moldavie. La seule différence c'est que les Juifs sont dix fois plus honnêtes, plus moraux, plus humains, que ces gens-là »". Au point que « si l'Orient devait choisir (ce à quoi, Dieu merci, l'on n'est pas encore arrivé) entre une domination grecque et une domination juive, il vaudrait mieux la seconde $»^{5}$.

3 Mais précisément, la domination grecque a existé dans les Principautés, pas «la seconde", ce qui fait une différence de taille. Dans sa comparaison cependant, Eminescu fait plus précisément référence au noyautage de l'élite et à l'accaparement des capitaux et des postes qu'à la figure du prince régnant d'origine phanariote. Ce qu'il attaque prioritairement dans le couple Juif-Grec est la position de parasite et de profiteur (deux termes récurrents), de déstabilisateur de la société et de la nation roumaine en formation. Ces deux figures ne jouent pourtant pas vraiment le même rôle, que ce soit dans la réalité de ces deux provinces comme dans la prose journalistique d'Eminescu, ni en général dans cette définition négative de soi par contraste avec l'étranger qu'a construit l'idéologie nationaliste roumaine à cette époque.

4 La perception des Grecs au XIX ${ }^{\mathrm{e}}$ siècle est encore fortement déterminée par l'épisode phanariote et tout ce qu'il charrie d'humiliation, de complexes et de frustrations nationales. Mais il apparaît aussi que cette critique virulente tient autant à des exigences du moment, issues du contexte politique propre à cette fin de siècle, qu'à une analyse historique objective de la question grecque dont le moment n'est pas encore venu. On tentera donc d'affiner les contours et les fonctions de l'image négative, rejetée, parée de tous les vices, qu'est celle du Grec, dans quelques textes de cette époque, en particulier chez Nicolae Bălcescu, Mihai Eminescu et Dumitru Drăghicescu. La confusion y est parfois grande entre la critique (historique) du régime phanariote et de ses effets catastrophiques (critique déjà ancienne) et celle des contemporains d'origine grecque, entre les arguments historiques, psychologiques, ethniques et politiques, et entre différentes fonctions de ces clichés qu'il faut distinguer dans le discours national : réaction épidermique contre l'étranger bouc émissaire? Rejet de l'Orient pour mieux valoriser la vocation occidentale de la Roumanie? Polémique politicienne instrumentalisant le passé phanariote des Principautés?

\section{L'épisode phanariote intégré dans « la marche de la révolution » nationale : Nicolae Bălcescu}

Bălcescu est celui qui lance le sujet avec son court texte de 1845 "Românii şi fanarioţii » [Les Roumains et les Phanariotes] $]^{6}$, destiné à introduire trois documents d'archives qu'il publie, dont deux sont des actes de princes valaques réclamant l'expulsion des Grecs hors de leur Principauté en 1631 et 1669. L'objet du texte est de 
souligner les "deux siècles de lutte» des Roumains contre les Phanariotes. Remarquons qu'avec ces « deux siècles » Bălcescu englobe une période plus longue que celle habituellement qualifiée comme « régime phanariote» (1711-1821) du fait que le processus commence avant l'arrivée au pouvoir des phanariotes, au moment où les princes autochtones commencèrent à s'entourer de fonctionnaires grecs (en 1613 avec Radu Mihnea). Bălcescu tient également à préciser le sens du mot "grec " dans son article par une note: le terme y désigne seulement les Grecs de Roumélie et de Constantinople, bref les phanariotes, et non les Grecs en général. À propos de ces derniers, l'auteur souligne au contraire la grandeur de la nation grecque actuelle, «que nous aimons et que nous respectons " et qui "se distingue des phanariotes qu'ellemême déteste et rejette $»^{7}$. Une référence à l'Essai sur les Phanariotes de Zallony (1824) vient appuyer la remarque. Voilà un Grec qui a lui-même dit : "les pages de l'histoire des provinces de la Moldavie et de la Valachie, si elle est écrite par une main ferme, impartiale et indépendante, ne seront remplies que des actes arbitraires de ces nouveaux despotes. Les malheureux habitants de ces provinces doivent frémir aux seuls noms de Fanar et de Fanariotes $»^{8}$. Cette distinction Grecs/Phanariotes est à noter, car elle sera de moins en moins de mise par la suite.

6 La « lutte » est le terme central, récurrent dans ces trois pages écrites au passé simple comme une longue saga, pleine de complots, de prises de pouvoir violentes, de princes renversés, de coups bas, d'empoisonnements, d'assassinats. La cause principale en est les « intrigues » des Grecs et ses effets, qui leur sont donc imputables, les « désordres » créés dans le pays, la mise à bas de la vieille constitution et la dégénérescence des traditions, le pillage des ressources, bref, « une situation pitoyable $»^{9}$. Ce texte du tout jeune historien (il a 25 ans) est plein de jugements de valeur: les Grecs (phanariotes) sont des «bandits ", des « misérables » ou des «scélérats », des «tyrans ». Contre eux, les réactions des princes ou boyards autochtones sont parfois "terribles», mais toujours "justes» et les Roumains morts dans cette lutte sont autant de "martyrs » « sacrifiés " pour la cause nationale. Le terme « national » n'apparaît pas (encore) dans ce texte pré-quarante-huitard, mais l'idée est bien là et la manière dont Bălcescu résume l'objet de son article est déjà typique de sa démarche : «[le] but est seulement de montrer les différentes phases de cette lutte de deux siècles entre les Roumains et les Phanariotes $»^{10}$. Il s'agit de découper l'histoire en phases pour mieux faire apparaître la continuité d'un processus orienté.

7 Les Phanariotes réapparaissent, cette fois-ci sous leur nom propre, dans le texte Question économique des Principautés danubiennes (1850), écrit en français, où ils s'entendent avec les ciocoï (parvenus constituant une bureaucratie d'arrivistes) pour, là encore, " dépouiller à qui mieux mieux, le pays " et "songer à mettre la main sur les libertés et les propriétés communales $»^{11}$. Mais le point de vue a changé. La question est devenue essentiellement sociale et non plus ethnique. Et les Phanariotes jouent ici un rôle particulier dans une histoire roumaine envisagée cette fois dans une perspective beaucoup plus large et où le point de vue moral a changé de niveau, passant du jugement des actes individuels à celui du sens de l'histoire. Les Phanariotes incarnent maintenant une "justice expiatoire » qui frappe les boyards roumains en punition de leurs "crimes", de la "spoliation" des petits paysans propriétaires qui a fait des principautés une terre régie par quelques grands propriétaires. «Les Phanariotes érigèrent l'anéantissement des boyards indigènes en système gouvernemental, comme les ancêtres de ceux-ci avaient organisé la dépossession territoriale des paysans ${ }^{12}$. » 
8 Cette idée-force de l'histoire-processus dans laquelle chaque phase prend son sens va apparaître particulièrement dans le texte Mersul RevoluŢiei în istoria românilor [La marche de la Révolution dans l'histoire des Roumains] (1850). L'épisode phanariote devient une étape de la longue «marche » vers l'avènement de la nation, au fil de laquelle les pics révolutionnaires ne sont que des manifestations plus visibles d'un processus profond et orienté.

L'histoire nous montre la révolution menant la nation roumaine de transformation en transformation dans la voie de cette loi universelle du développement historique des nations : l'accession du plébéianisme au pouvoir ou comme nous le formulons, la réintégration du roumain, l'esclave, le serf du Moyen-Âge, dans ses droits d'homme, de citoyen et de nation. En vérité, ce coup d'œil historique nous a montré que l'État, de princier [domnesc] ou absolu, est devenu seigneurial [boieresc] ou aristocratique, puis phanariote ou bourgeois [orăşenesc] (bürger), puis parvenu [ciocoiesc] ou bureaucrate et il est maintenant en voie de se faire roumain ou démocratique ${ }^{13}$.

Ce résumé synthétique est remarquable et typique de Bălcescu. Les stades de l'histoire y sont désignés par une double série de termes faisant correspondre le schéma roumain (domnesc, boieresc, fanariot, ciocoiesc, românesc) à des catégories occidentales (absolu, aristocratique, bourgeois, bureaucrate, démocratique), à un schéma considéré comme général, "normal ", dans les philosophies de l'histoire du xix siècle. Le modèle matérialiste marxiste n'est pas loin non plus. «L'accession des phanariotes au pouvoir eut en tout point les caractères de l'ascension du monde urbain en Europe occidentale $»^{14}$, les Grecs remplissant en Roumanie la fonction qu'eut la bourgeoisie dans les sociétés occidentales. Dans cette optique, la lutte entre groupes nationaux (roumains contre phanariotes étrangers) tend à devenir lutte entre des classes (bourgeoisie contre boyards).

10 Le schéma rappelle celui des philosophies allemandes de l'histoire du $\mathrm{XIX}^{\mathrm{e}}$ siècle également par son moteur dialectique, de progression par dépassement des contradictions et des luttes. Les phanariotes sont toujours représentés de manière aussi négative, comme des "hommes corrompus, misérables", "étrangers à leur propre peuple et ennemis de celui-ci », prenant le pouvoir par la ruse et la tromperie, et leur règne est "un siècle d'oppression et de pillage, de corruption et de dégradation " synonyme "d'affaiblissement » de la nation roumaine. Mais cette phase de régression dans la marche de la nationalité était en réalité nécessaire. Nécessité morale tout d'abord, comme on l'a vu dans le texte précédent, les phanariotes représentant une "punition" pour les boyards coupables d'avoir asservi le peuple. Il fallait que « cette classe d'apostats " soit à son tour asservie, qu'ils soient renvoyés à leur condition première (car ils sont sortis du peuple) et « pour que la punition et la honte soient plus grandes ", il fallait qu'ils le soient par une classe d'asservis, et d'asservis étrangers. Donc une expiation pour "l'état seigneurial [boieresc]", une réponse à l'étape précédente en même temps qu'une préparation pour la suivante. Car c'est du négatif que sort le progrès : la constitution de Mavrocordat, si elle est aussi néfaste que l'ordre précédent, a des effets profonds, elle "révolutionne les états roumains»: en anéantissant les libertés des boyards, qui disparaissent (en tant que classe dominante), c'est le servage qui disparaît également et le sentiment national qui se réveille, alimenté par l'infâme politique des phanariotes. Lorsque « le mal » est à son comble, la révolution (1821) balaie ce régime et l'on passe à la phase suivante. "Ainsi, les phanariotes aussi, sans le savoir et sans le vouloir, accomplissent une mission 
providentielle et aident le progrès $»^{15}$. On retrouve là le vieux schéma hégélien, sans doute passé par Michelet et Quinet, qui trouve son application dans le cas roumain : le travail du négatif comme moteur de l'histoire et la «ruse de la Raison", sorte de providence cachée, pour expliquer le progrès.

11 Cette interprétation englobante de l'historien-philosophe, capable de dépasser le cliché qu'il contribue à construire pour voir un sens général de l'histoire, est très propre à la formation intellectuelle de Bălcescu et à son parcours de révolutionnaire idéaliste. Ce n'est évidemment pas cette vision « dialectique » des phanariotes qui s'impose. Encore moins une hypothétique figure positive de l'étranger, même dans un contexte révolutionnaire et à une époque où commence à circuler l'internationalisme. Il n'y a que dans un texte aussi exalté que la Proclamation d'Islaz de juin 1848 que l'appel à la fraternité internationale peut se formuler :

Citoyens en général, prêtres, boyards, soldats, commerçants, artisans de toute catégorie, de toute nation, de toute religion, qui vous trouvez dans la capitale et dans les villes, Grecs, Serbes, Bulgares, Allemands, Arméniens, Israélites, prenez les armes pour le bon ordre et pour aider à cette grande action. La patrie est la nôtre et la vôtre. Il vous plaît d'y habiter et elle vous accueille. Le vieux système ne vous a pas appelés à la table publique. À partir d'aujourd'hui nous avons une seule table, ensemble, un banquet fraternel nous appelle, nous aurons tous les mêmes droits ${ }^{16}$.

Heliade-Rădulescu qui en est le rédacteur principal ne va pas tarder à tenir des propos xénophobes et Brătianu, l'un des meneurs du mouvement et futur chef du gouvernement, opte rapidement pour une conception nationale étroite qui se réfère au neam ("peuple » au sens ethnique, à distinguer du "popor ", au sens plus politique et social, français) et à la théorie des races de Blumenbach. L'antisémitisme d'un Simion BărnuŢiu (meneur du mouvement quarante-huitard transylvain) est notoire et la xénophobie à ses différents degrés, en cette époque de nationalisme virulent, est la chose du monde la mieux partagée, entre libéraux et conservateurs.

\section{Le Grec et le Juif : deux incarnations de la xénophobie des nationalistes roumains (Mihai Eminescu et Dumitru Drăghicescu)}

13 Le grand moment d'élaboration de l'idéologie nationale qui culmine en 1848 s'est fait dans le cadre d'une réorientation vers l'Occident de la culture roumaine. Les phanariotes en ont été, au moins indirectement, l'un des facteurs par leur rôle d'intermédiaires dans l'expansion de la culture occidentale, française notamment. Mais avant que l'historiographie mette en lumière ce phénomène, cet apport positif de la présence grecque dans les Principautés - par le biais des précepteurs et de leurs bibliothèques - les Grecs incarnent avant tout l'Orient et Byzance. Or la découverte, aussi exaltante que traumatisante, de l'Occident et corrélativement du retard roumain déclenche une prise de distance forte, de plus en plus violente, vis-à-vis de cet Orient accusé d'être responsable de l'état d'arriération des Principautés. En témoigne le cliché de la «barbarie orientale » que l'on retrouve dans les textes de l'époque ${ }^{17}$. Ces clichés sont systématisés et synthétisés par le sociologue Dumitru Drăghicescu, vulgarisateur en Roumanie des thèses de psychologie des peuples dont il propose une application à l'histoire de son pays dans son ouvrage de 1907 Din psihologia poporului român [Sur la psychologie du peuple roumain]. La Roumanie se trouvant par sa position géographique au 
point de rencontre des deux mondes, oriental et occidental, elle fut le lieu et l'objet d'une double influence. Malheureusement pour elle, l'Orient l'a marquée en premier et retardé d'autant la saine influence occidentale. Cet héritage dont il faut se débarrasser est arrivé indirectement par les Slaves et l'église et directement par les Grecs et les Turcs, estime l'auteur ${ }^{18}$, qui le résume ainsi: "paresse de l'esprit, négligence et fainéantise du corps, passions lascives, corruption, propension à faire traîner les affaires en longueur, manque de confiance en soi, passivité, résignation, croyance dans le destin, la chance et la providence, fatalisme $»^{19}$.

Cette réorientation vers l'Occident, ce sont principalement les Grecs qui en ont fait les frais, plus que les Turcs, et, en cette fin de $\mathrm{XIX}^{\mathrm{e}}$ siècle roumain, sans doute même plus que les Juifs. La figure du Turc paraît en effet moins dangereuse, car plus radicalement autre : plus éloigné et surtout musulman. Il est plus l'objet, comme le remarque Lucian Boia, d'images d'Épinal, d'un orientalisme exotique ${ }^{20}$. Le Grec au contraire est dangereux, car doublement proche du roumain: orthodoxe, et installé depuis longtemps sur le territoire des Principautés. «Infiltré », dit Eminescu, qui relève cette double proximité, l'une étant la cause de l'autre: «Ce que sont les Juifs pour la Moldavie, ces métèques le sont pour la Valachie, eux qui, grâce à l'identité de religion, ont su s'infiltrer parmi les Roumains, les abuser pour finalement les dominer $»^{21}$. C'est une explication facile pour la situation difficile des Principautés : à chacune son bouc émissaire venu de l'étranger et installé sous la forme du parasite-profiteur. Mais le parallélisme Grecs-Juifs proposé par Eminescu doit être discuté. Si les effets de leur présence sur l'état des Principautés semblent être comparables en termes de degré de catastrophe (Eminescu fournit des chiffres sur la pauvreté, la baisse démographique, l'argent détourné...), il y a en quelque sorte un partage des tares et des torts entre ces deux communautés. L'histoire de leur immigration est différente, de même que les motifs et les stratégies de leur "infiltration », pour reprendre l'idée des auteurs de l'époque.

15 La présence des Grecs en nombre est ancienne et déborde largement, tant chronologiquement que par leur empreinte sur la société roumaine, le régime des princes phanariotes. La prise de conscience de ce phénomène explique d'ailleurs que le terme de «Grecs » remplace de plus en plus souvent celui de « phanariotes » dans les textes, voire peut désigner les populations trans-danubiennes en général (comprenant aussi les Bulgares notamment) dont la présence est liée au rattachement des Principautés à l'Empire ottoman. Notons l'existence du terme péjoratif grecotei pour les désigner, fréquent dans les articles d'Eminescu (à côté des bulgăroi, bulgares et des jidani, juifs). Cette migration ancienne, avant d'être le fait du régime phanariote, s'est faite, comme le résume Drăghicescu dans le chapitre de son livre explicitement consacré aux Grecs, par le biais de l'église, du commerce et du clientélisme ${ }^{22}$. Paradoxalement, l'orthodoxie des Grecs est un tort retenu contre eux chez les deux auteurs, alors que la différence de religion n'est pas l'argument principal retenu contre les Juifs dans les textes de cette époque (contrairement aux années 1920-1930). La religion en elle-même intéresse assez peu Eminescu et les penseurs de cette époque. Mais c'est un facteur essentiel par ses effets : les moines grecs se sont fait accepter et admirer pour leur piété, ils ont fondé des monastères et sont de ce fait détenteurs de grandes propriétés. La pratique religieuse des Grecs a facilité en général leur intégration (leur "infiltration») dans la société roumaine. Venus d'un empire musulman, ils se sont « réfugiés » dans les Principautés « sous le masque de la religion, 
facile à porter ", écrit Drăghicescu après Eminescu qui donne à plusieurs reprises l'exemple du patriarche Nifon, appelé par le prince Radu le Grand et qui, « au lieu de s'occuper de religion, s'est mis à faire de la politique, à créer son parti parmi les boyards, à aiguiser et envenimer " les rivalités entre les familles ${ }^{23}$. La Constitution de 1866 ne les privant pas des droits politiques (seuls les étrangers de rite chrétien peuvent prétendre à la citoyenneté, dit l'article 7) ni de la possibilité d'acheter des terres, leur exemple est abondamment exploité par Eminescu pour argumenter son refus d'accorder ces droits aux Juifs. Mais les Grecs sont surtout accusés d'avoir constitué l'avant-garde, l'entourage et la longue suite des princes nommés par la Porte, dont ils tenaient les cordons de la bourse et auprès de qui ils détenaient de nombreuses charges administratives, avant de fournir directement des prétendants aux trônes des Principautés. "Des Grecs de Constantinople et des Principautés faisaient alliance, constituaient avec leurs économies un capital important, se groupaient autour d'un phanariote influent à la Porte et achetaient le trône de l'une ou l'autre des Principautés $»^{24}$. Bref, ils représentent la collusion du pouvoir et de l'argent, qui plus est sous la bannière du sultan turc.

L'immigration juive, elle, a surtout été ressentie après la vague des Galiciens arrivés en Moldavie du XIX ${ }^{e}$ siècle. Si elle est ressentie comme dangereuse, c'est parce qu'elle est vécue comme brutalement «invasive » et qu'elle atteint toutes les classes, jusqu'aux plus basses, celles des campagnes, alors que les Grecs sont surtout présents en ville et dans les fonctions de l'État. De ce fait leur présence et leur insertion dans la société (Eminescu dirait leur forme de " parasitisme ») ne peut être la même. Les « nuisances " qui leur sont reprochées sont autres. À côté de quelques grands banquiers et entrepreneurs industriels que l'on accuse de monopoliser le capital national, ce sont leurs fonctions de fermiers et d'usuriers qui sont détestées ou, plus bas dans l'échelle sociale, de tenanciers de débits de boissons. Ils s'attachent ainsi le bas peuple par l'usure, l'exploitent sur des terres qui ne leur appartiennent pas et répandent l'alcoolisme, bref ils sont responsables de la misère paysanne, affirme Eminescu - sur la base de statistiques détaillées en matière de démographie, de santé, de pauvreté - et en cela c'est la nature même du paysan roumain, dépositaire de l'identité nationale, qui est atteinte.

Le réquisitoire d'Eminescu contre les Juifs est fondé sur une argumentation essentiellement économique et nationaliste quand celui réservé aux Grecs est indéniablement de nature raciste. L'ancienneté de leur présence et le "privilège " d'avoir «régné» en la personne des princes phanariotes permettent d'en faire facilement les responsables de tous les vieux problèmes des Principautés. Car il y a «l'identité et l'atavisme du caractère » :

Si le bon ordre et l'organisation de ces pays se sont perdus, si nous avons perdu des provinces, si nous avons facilement abandonné les anciennes et bonnes habitudes, si la corruption et la lâcheté se sont introduites dans les classes de la vieille société roumaine, il y a toujours eu un Grec ou un groupe de Grecs à l'origine de ces maux. Et cette nation qui en vieillissant a perdu toutes ses qualités, ne conservant que ses vices antiques, qui au cours du Moyen-Âge a corrompu l'architecture, la musique, la peinture, ce peuple qui a envenimé la vie des peuples voisins, qui les a trahis auprès des Turcs à seule fin de conserver sa position hiérarchique, qui a volé un cinquième des terres roumaines, cette nation aux instincts innés de fourberie et de brigandage devrait être à la tête de l'Orient ${ }^{25}$ ? 
Selon Drăghicescu, les Grecs - de Byzance - ont même contaminé les Turcs par leur corruption morale et sont donc responsables également de la décadence de l'Empire ottoman ${ }^{26}$.

19 La description de leur duplicité et de leur aptitude à la bassesse qui sont autant de vertus dans ces marges de l'Empire ottoman en décomposition alimente nombre d'articles d'Eminescu chez qui cela devient un thème obsessionnel dans les colonnes du journal Timpul au début des années 1880 . On reste médusé aujourd'hui par la violence des propos, profondément marqués par les thèses racistes qui traversent l'Europe de l'époque. Ce ne sont ni la langue ni la nationalité qui justifient ce rejet, mais bien «les qualités morales et intellectuelles de la race » se reflétant dans des qualités physiques qui sont autant de "signes" permettant de reconnaître ces êtres "voués à la dégénérescence", faute d'être condamnés à la pendaison qu'ils méritent ${ }^{27}$. L'auteur dresse un portrait épouvantable de ces populations comparées à des alluvions boueuses laissées par les débordements de byzantinisme et de balkanisme au-delà du Danube.

Et comment n'en serait-il pas ainsi ? Cette plèbe est recrutée à Byzance, de l'empire grec d'Orient. Il faut se représenter l'histoire de cet empire, ces mille ans de crimes honteux, de misère, de démagogie, il faut se rappeler que c'était l'empire dans lequel les pères violaient leurs filles, le fils arrachait les yeux du père, dans lequel on se moquait du mariage et où l'âme comme le corps étaient à vendre, et l'on comprendra alors que les causes qui ont persisté un millénaire ne peuvent pas ne pas s'être incarnées, matérialisées, dans la race d'hommes qui vivait là-bas. La loi de la causalité est absolue, ce qui s'est produit un millier de fois à Byzance et jusqu'à aujourd'hui est nécessairement passé dans la constitution physique et morale de ce peuple, s'est enraciné sous forme d'un regard perfide, torve et myope, dans une physionomie de chèvre, dans la tendance à être bossu. À la perfidie du regard correspond le daltonisme intellectuel à l'égard de toute qualité morale, que ce soit l'honneur, la dignité ou la vérité; à la déformation physique correspond la déformation morale. Car ce qui ne ment jamais, c'est bien la nature ${ }^{28}$.

20 Il faut dire qu'Eminescu trouve dans la littérature "scientifique " de l'époque des références à citer, comme l'ethnologue français Guillaume Lejean qui a écrit une Ethnographie de la Turquie d'Europe (1861) et son confrère allemand, Rudolf Henke, auteur de Rumänien, Land und Volk (1877), qui fait le même classement en matière d'immoralité, où le Grec surpasse le Juif : les Grecs fournissant à la fois les contingents de criminels et d'avocats, les crimes ne sont ni découverts, ni punis. Pour le fonctionnaire grec, tout est à vendre : il commence par une caisse d'oranges et finit multimillionnaire. D'ailleurs on ne trouve pas dans le dictionnaire du néo-grec le mot de "moralité ", etc. Bref, tous des frères de Scarvuli, conclut Eminescu, faisant référence à un scandale de l'époque, une affaire de pédophilie qui a fait la une des journaux roumains en $1880^{29}$.

21 Le problème le plus grave pour Eminescu est la « contamination » profonde qui s'opère par les croisements de peuples et les effets qui en découlent, selon la thèse de la transmission des caractères acquis alors en vogue. Les Grecs de 1880 ont hérité du patrimoine dégénéré des Byzantins et des Phanariotes et ils le transmettent aux populations avec lesquelles ils sont en contact, corrompant ainsi la nature encore intacte et forte des peuples encore "jeunes». "Malheur au peuple jeune, avec ses instincts généreux, son intelligence malléable et ouverte à la vérité lorsqu'il entre en contact avec cette lie de l'humanité, ces restes de vieux peuples passés par toute la misère d'une civilisation morte $\aleph^{30}$. De ce point de vue, les croisements avec les Juifs sont moins problématiques, s'agissant d'une race sinon plus jeune, du moins plus noble, 
ils sont même considérés comme la seule façon pour eux de s'intégrer un jour au peuple roumain, par mariages mixtes. Car contrairement aux Grecs qui se "roumanisent " facilement, mais superficiellement - pour des raisons d'intérêt - en apprenant la langue et en investissant l'appareil de l'État, les Juifs gardent farouchement leur langue, leur culture et leurs traditions. Les Grecs qui acquièrent facilement la nationalité roumaine font semblant d'être Roumains et se présentent comme tels, les Juifs qui la réclament sans l'obtenir ne veulent pas être Roumains, selon Eminescu. Ces deux situations sont également nuisibles pour les Principautés.

Mais Eminescu lui-même ne mène pas cette comparaison entre Juifs et Grecs, dont les cas sont quasiment toujours traités séparément. Son antisémitisme et sa xénophobie principalement anti-grecque correspondent globalement à des phases distinctes de son œuvre de "publiciste ", mais aussi de l'histoire roumaine qui lui est contemporaine. Les descriptions consternées de l'état de la Moldavie sous l'emprise des Juifs et le déchaînement qui s'ensuit remplissent les pages de Timpul dans les années 1877-1879, au moment des débats sur la révision de l'article 7 de la constitution imposée par les grandes Puissances au congrès de Berlin comme condition de la reconnaissance de l'indépendance roumaine. La phase violemment anti-grecque est postérieure. Ces deux moments journalistiques peuvent aussi correspondre à une géographie de la carrière d'Eminescu, même s'il y a décalage : ses articles de fond des années 1876-1879 doivent beaucoup à la période passée à Iaşi au journal Curierul de Iaşi et généralement à sa connaissance de sa région natale, la Moldavie du Nord. Son déménagement à Bucarest lui donne ensuite la possibilité de faire connaissance avec la société valaque et de suivre au plus près le feuilleton politique de la capitale. L'évolution des centres d'intérêt de ses articles en porte la marque.

L'argument du cadeau empoisonné reçu de l'extérieur pour expliquer ses propres défaillances est un vieux sophisme, fréquent chez les Roumains qui ont une longue pratique de l'histoire du peuple victime, victimes des entreprises de l'étranger (Turcs, Russes, Allemands, Juifs, Autrichiens, Hongrois, Soviétiques...). Pour ce qui concerne les Grecs l'ouvrage déjà mentionné de Dumitru Drăghicescu est exemplaire. C'est la première synthèse roumaine des questions de psychologie des peuples débattues dans toute l'Europe intellectuelle. Eminescu connaissait déjà bien Lazarus et Steinthal (inventeurs du concept de Völkerpsychologie) et il faut aussi citer Constantin RădulescuMotru, philosophe et psychologue formé en Allemagne auprès de Wilhelm Wundt et fondateur de l'école roumaine de psychologie et de psychologie sociale. Dans l'ouvrage de Drăghicescu, l'influence des Turcs et des Grecs sur la psychologie des Roumains constitue un chapitre à part entière constituant une bonne synthèse de tous les développements, jugements de valeur, clichés et condamnations rencontrés dans les articles d'Eminescu trente ans auparavant. Mais la brutalité du propos choque d'autant plus qu'il ne s'agit plus d'articles de presse dont les analyses sont motivées par l'actualité quotidienne et tributaires des passions du moment, mais d'un ouvrage "scientifique » dans lequel on pourrait attendre un ton posé et plus nuancé. Or, on y relit que la « catastrophe grecque qui frappa si cruellement notre pays » fut l'œuvre de "véritables parasites", d'" envahisseurs" à la "vanité perfide", une "vermine grecque ", le "chiendent du Phanar ", que cette œuvre consistât en "destruction", " asservissement ", " corruption ", " persécutions ", par des procédés d'" infiltration ", de "tromperie » et de "spéculation », de "basse flagornerie » ou de "calomnie », des "intrigues", tous les "pièges perfides du Phanar", véritable «maladie", «putréfaction morale » qui a «contaminé » et « intoxiqué » les Principautés, bref, que 
ce fut une "plaie fatale $»^{31}$. On est presque surpris de trouver un ou deux éléments positifs dans ce texte : l'auteur note que l'adoption de la langue grecque par les élites a permis de se débarrasser plus vite du slavon et que la petite aristocratie roumaine s'est dégrossie au contact des Grecs, a gagné un peu de culture et de "raffinement». Malheureusement, une culture de la «tromperie » et un raffinement plus propre aux peuples décadents... Drăghicescu reprend aussi l'analyse socio-économique entamée par Bălcescu et Eminescu pour conclure que le gouffre villes-campagnes typique de la société roumaine s'explique de façon simple par l'immigration grecque. Cette communauté étrangère ayant dès le début visé les fonctions avantageuses de l'administration, du commerce, de la finance et du pouvoir, elle a infiltré les grandes familles de boyards et fourni les plus gros contingents de la population urbaine quasi inexistante avant sa venue, quand les campagnes restaient majoritairement paysannes, roumaines et pauvres. Ethnies, classes et milieux se recoupent alors en strates qui se correspondent: «la différence ethnique entre les nobles et le peuple s'accentue fortement [au XVIII ${ }^{\mathrm{e}}$ siècle] et de là le fait qu'aujourd'hui encore le peuple roumain présente l'aspect de deux races totalement étrangères l'une à l'autre, deux races superposées ou juxtaposées qui n'ont rien en commun : les citadins et les villageois $~_{32}$. L'expression de « races superposées » ne peut pas ne pas évoquer à nouveau Eminescu qui revendique la paternité de la théorie des «couches superposées » pour expliquer l'évolution et le fonctionnement de la société roumaine au XIXe siècle. Il faut y revenir.

\section{Les « nouveaux phanariotes ». Le recyclage de la critique des phanariotes et sa mobilisation politique en 1880}

Le contexte politique des années 1880 contribue à expliquer la phase anti-grecque d'Eminescu, de même que l'épisode du Congrès de Berlin motivait ses articles antijuifs de 1879. Cette véritable explosion de fureur correspond au moment où le "long règne " du premier ministre I.C. Brătianu (1876-1888) est bien installé et appuyé sur le parti libéral dont un groupe en particulier, celui de C.A. Rosetti, le "parti rouge", est désigné comme celui des "nouveaux phanariotes " ${ }^{33}$ par Eminescu. Sa critique vise donc prioritairement une fraction de la population d'origine grecque de Roumanie accusée d'être une faction du monde politicien de Bucarest.

Remarquons au passage que l'expression de "parti phanariote ", qui désignait chez Bălcescu celui des boyards conservateurs restés fidèles aux Phanariotes historiques et en même temps "philo-tsaristes" (au nom de la solidarité orthodoxe), par opposition au jeune " parti national » des quarante-huitards libéraux, va désigner chez Eminescu précisément certains de ces anciens quarante-huitards, anciens compagnons de Bălcescu. C'est bien le signe que la critique des "phanariotes » est un passe-partout politique largement recyclable.

La thèse d'Eminescu en l'occurrence est que, derrière une apparente évolution historique qui aurait profondément modifié la situation politique des Principautés, la situation de la Roumanie en 1880 est tout à fait comparable à celle du XviII siècle lorsqu'elles étaient soumises au régime phanariote. Ce sont des Grecs qui les gouvernent, en utilisant les mêmes procédés de dissimulation et de corruption, dit en substance Eminescu. 


\begin{abstract}
À Bucarest et dans les villes du bord du Danube est apparu un élément ethnique complètement nouveau et hybride qui fournit la génération actuelle de nos gouvernants. Ils sont ce qui reste des vauriens qui suivaient Pasvantoglu et Ypsilanti et les résidus nombreux des cavaliers de l'industrie du Phanar. C'est de cette nouvelle lignée que font partie des gens comme Giani, Carada, C.A. Rosetti, Phyrekydis, Serurie, etc. Toute cette écume de fanariotes «novissimes » qui se sont installés dans le pays depuis 50-60 ans, forme naturellement l'élément de dissolution, la démagogie de la Roumanie ${ }^{34}$.
\end{abstract} fréquentes au point de devenir un tic d'écriture de l'auteur. Le premier visé de cette liste est C.A. Rosetti, ancien quarante-huitard et bras droit de I.C. Brătianu, au sujet duquel Eminescu tient à dissiper une confusion dont les libéraux joueraient pour légitimer l'autorité de leur chef : son père était grec et il n'a rien à voir avec la famille Rosetti de Moldavie, « famille historique $»^{35}$. Eugen Carada, fondateur et directeur de la Banque Nationale, est une autre cible favorite, parfois désignée allusivement par l'expression «quatre classes d'école primaire et un cours de violoncelle », symbolisant l'arrivisme et le clientélisme mal dissimulés derrière une éducation très lacunaire et un semblant de raffinement bourgeois. Lorsqu'on reproche à Eminescu de faire une fixation sur quelques noms et de s'en prendre sans cesse à Pherekydis, il réplique avec la liste des «convives» de C.A. Rosetti au grand «banquet» du pillage budgétaire, prenant plaisir à démasquer derrière les rares noms aux consonances roumaines l'origine russe, bulgare, bosniaque ou tsigane du personnage ${ }^{36}$.

Plus sérieusement, sa thèse d'un néophanariotisme roumain s'appuie sur une série de «petite[s] leçon[s] d'histoire $»^{37}$ qui alimentent ses articles accusateurs. Il s'agit déjà, pour justifier le lien avec les phanariotes historiques, de réinsérer ces "nouveaux phanariotes » dans une continuité historique, et Eminescu fait lui aussi la chronologie d'une «lutte » entre «l'élément autochtone » et "l'élément immigré » qui rappelle le texte du jeune Bălcescu, mais selon un schéma plus grossier et simpliste :

En 1700 c'est l'élément immigré qui l'emporte avec l'instauration du régime phanariote.

En 1821 la réaction de l'élément autochtone se déclenche et avance victorieusement et par assimilation jusqu'en 1866.

Le 11 février 1866, c'est à nouveau l'élément immigré qui l'emporte ${ }^{38}$.

D'ailleurs on observe après cette date les mêmes phénomènes que pendant la période phanariote (1700-1821), note Eminescu: baisse de la population, appauvrissement, recul du niveau d'éducation et hausse de la corruption. Mais le conflit est devenu explicitement une lutte de races ${ }^{39}$, conformément aux théories en vogue à l'époque pour une part importante de l'Europe intellectuelle. Concrètement, il s'agit d'une immigration des années 1830-1840, mais aussi des restes des troupes d'Ypsilanti et surtout des domestiques des boyards autochtones, affirme Eminescu sur la foi de documents historiques... de source grecque puisqu'il s'agit en particulier d'un discours tenu par Ypsilanti lui-même à ses compatriotes des Principautés pour les enrôler dans sa lutte hétairiste. Or voici en quels termes il s'adressait à eux :

Vous, mes amis patriotes, tous autant que vous êtes, que les événements et le besoin a traînés sur ces terres de Moldavie et de Valachie, contraints par les hasards hostiles, vous en êtes arrivés à prendre place jusque derrière les calèches des boyards autochtones de ces pays, ce qui est une honte et une infamie encore jamais rencontrées parmi les habitants de l'Hellade. Et tant que vous resterez sourds à cet appel sacré de la patrie et que vous voudrez rester les serviteurs des boyards de ces pays, en vous tenant derrière leurs calèches, sachez qu'à partir de ce moment vous 
ne pourrez plus porter les armes et qu'il vous faudra vous revêtir de l'habit des

habitants d'ici et vous installer au sein de leur nation ${ }^{40}$. plus. C'est que ces deux immigrations ne sont que les deux manifestations les plus frappantes du phénomène général qui, selon Eminescu, explique la situation des Principautés en cette fin de $\mathrm{xIx}^{\mathrm{e}}$ siècle et qu'il formule comme sa théorie des « couches superposées ", à laquelle Drăghicescu faisait allusion :

Il faut distinguer: nous avons d'un côté la race roumaine, avec son passé, race identique dans tous les pays qu'elle habite, peuple honnête, brave, capable de vérité et de patriotisme. Et nous avons au-dessus de ce peuple une couche superposée, une sorte de sédiment d'escrocs et de prostituées, qui vient du mélange de tous les déchets de l'orient et de l'occident, incapable de vérité et de patriotisme, la race des Carada, que les Moldaves confondent avec les Valaques ${ }^{44}$.

«Ce qu'ont fait les Carada d'il y a 60 ans ", commente Eminescu, désignant par là les porteurs de noms grecs comme autant de descendants de domestiques qui ont pris l'habit, se sont déguisés en Roumains pour mieux s'installer. Ce faisant, ils se sont faits traitres à leur patrie. Mais c'est là leur nature de Grec, explique Eminescu, et «l'histoire de Tudor et de Cuza illustrerait cette théorie ", ajoute-t-il ${ }^{41}$. On peut les interpréter en effet comme deux histoires de trahison, la première par Ypsilanti faisant arrêter et exécuter en 1821 son ancien allié Tudor Vlădimirescu, la seconde par Rosetti, membre de la «monstrueuse coalition" entre libéraux et conservateurs qui renversa Cuza en 1866, un Rosetti devenu monarchiste après avoir été républicain en 1848.

la lutte de l'intérieur, investir de nouveau l'appareil politique et administratif, mais d'une manière plus dissimulée et donc plus efficace, qu'Eminescu s'est fait un devoir de démasquer : la stratégie de ces Grecs est de se faire passer pour plus Roumains que les Roumains par leur engagement auprès de la nation, en vérité totalement intéressé. Lorsque C.A. Rosetti et Carada fondent un journal, ils l'appellent Le Roumain [Românul] ! s'emporte Eminescu qui rebaptise souvent la publication «pseudo-românul» ou «journal des phanariotes $»^{42}$. La genèse du "parti rouge » qu'il propose est l'histoire d'une infiltration dans les rangs du parti "national " au moment même où la nation s'affirme (dès 1848 pour C.A. Rosetti) et surtout où se construit un appareil étatique dont il est ainsi aisé de s'approprier les postes clés.

Le parti rouge, avec son organisation pourrie, avec sa soumission absolue à un vieux phanariote, n'est pas un parti politique, mais une société secrète, ennemie de l'État, analogue à la Mafia et à la Camorra, ressemblant beaucoup aux ramifications de l'Alliance israélite. [...] Il travaille pour anéantir tout ce qui est roumain, pour substituer partout au Roumain le phanariote ou du moins des individus aussi corrompus que les phanariotes, afin de démembrer et d'empoisonner le peuple roumain ${ }^{43}$.

La théorie en question est ici ramenée à sa plus simple expression, raciste, mais elle a une interprétation économique longuement développée dans d'autres articles: les classes superposées sont celles qui vivent du travail de celles d'en dessous (la paysannerie roumaine qu'elles exploitent) sans "compenser» ce travail par leur propre contribution puisqu'elles sont improductives, constituées d'une armée de fonctionnaires profiteurs, de journalistes incultes, d'avocats, de gratte-papier administratifs et bien sûr de députés et de ministres, une "plèbe de la plume » et tout un appareil administratif et financier qui met l'état en faillite. C'est le diagnostic d'une profonde perturbation de l'organisme du pays, de sa vie économique, dont le bon 
fonctionnement est conçu, dans l'esprit d'Eminescu, sur des bases proches de la thèse des physiocrates. Pour lui comme pour ces penseurs français de la fin du XVIII ${ }^{e}$ siècle, les paysans sont la seule classe " positive », celle qui satisfait aux besoins vitaux et produit véritablement de la valeur en exploitant la nature. Ce n'est qu'à partir de son surplus de production que peut se construire toute la civilisation d'un pays, sa vie politique, culturelle, intellectuelle et spirituelle. Commencer par là c'est au contraire prendre les choses à l'envers et se condamner à en rester à ce que Maiorescu appelle les «formes sans fond » de la société roumaine. Eminescu qui a beaucoup fréquenté le cercle et les écrits de Junimea, s'est imprégné de la critique maiorescienne et sa thèse des « couches superposées » en est une variation dont les deux formulations, économique et raciste, lui sont effectivement personnelles. Cette théorie lui permet de réunir Grecs, Juifs et toutes les autres minorités, notamment trans-danubiennes (Bulgares, Turcs, Tsiganes). Mais les Grecs semblent y conserver une place "privilégiée » en matière de clichés : "Grecs laboureurs ou artisans, vous n'en trouverez guère en Roumanie. Fonctionnaires et spéculateurs, autant que de cheveux sur la tête. [...] Dans un siècle, le peuple latin des bords du Danube ne sera plus qu'une légende chantée dans la langue du Phanar ${ }^{45}$.

En cette fin de XIX ${ }^{e}$ siècle secouée et déchirée par les nationalismes, la figure du Grec est devenue dans les Principautés roumaines l'objet de toutes les projections xénophobes, de tous les fantasmes et de toutes les peurs, car elle conjugue celle, historique, du phanariote avec celle de l'étranger en général, celle de l'immigré avec celle du parvenu, se situant en quelque sorte à la «bonne distance » pour que s'exercent la haine et les clichés, celle d'un Autre assez proche pour être bien connu et insupportable: le Grec représente plus ou moins inconsciemment cet héritage balkanique que certains Roumains, jusqu'à aujourd'hui, n'aiment guère à se rappeler.

Il a cependant assez brutalement disparu du discours public au début du $\mathrm{xx}^{\mathrm{e}}$ siècle. Certes, l'historien (pourtant nationaliste) Nicolae Iorga en mettant en lumière l'héritage balkanique et notamment byzantin dans les Principautés, contribue à rééquilibrer la manière dont les Grecs sont considérés. Mais c'est aussi que les urgences historiques ont changé. La question d'Orient n'est pas loin de se clore. Les guerres balkaniques font émerger dans le débat public roumain (et européen) la vie, la mission et la lutte nationales des Grecs contre les Ottomans. Et surtout, de l'autre côté, sur l'autre marge de leur territoire, les Roumains sont de plus en plus préoccupés par la question transylvaine. Plutôt que de se focaliser sur les étrangers à l'intérieur de leurs frontières, ils ont maintenant les yeux rivés sur leurs compatriotes encore à l'extérieur et ils pressentent que la confrontation européenne que l'on sent venir sera décisive. La question grecque n'est plus une question d'avenir pour les Roumains, les enjeux sont maintenant ailleurs. 


\section{BIBLIOGRAPHIE}

Bălcescu Nicolae, (1940) «Question économique des principautés danubiennes », Opere, tomul I, partea a II-a, Scrieri istorice, politice şi economice, Bucureşti :FundaŢia pentru literatură şi artă

"Regelui Carol II".

Bălcescu Nicolae, (1953) «Mersul RevoluŢiei», Opere vol. I, Studii şi articole, Bucureşti :Ed.

Academiei Republicii Populare Române, p. 307-313.

Bălcescu Nicolae, (1953) «Românii şi fanarioţii», Opere vol. I, Studii şi articole, București :Ed.

Academiei Republicii Populare Române, p. 69-78.

Boia Lucian, (2011), Istorie şi mit în conştiinŢa românească, Bucureşti : Humanitas.

Cazacu Matei, (1993) «Eminescu Şi istoria naţională», Europa. Balcanica - Danubiana - Carpathica, 1, Annales. Cultura - Historia - Philologia, Budapest, p. 163-173.

Drăghicescu Dumitru, (1995), Din psihologia poporului român [1907], Bucureşti : Albatros.

Eminescu Mihai, (1999-2000) Opere, Publicistică (vol. III, IV, V) București : Academia română, Univers enciclopedia, 1999-2000.

Murăraşu Dumitrou, (1932), NaŢionalismul lui Eminescu, Bucureşti : Ed. Bucovina.

Constantinescu Gabriel, (2012), “'The horrible crime' of Scarvulis (1880). Ethnical Stereotypes regarding the Greeks in Romania during the latter of the 19th Century" in Analele UniversităŢii din Craiova, Serie Istorie, Anul XVII, nr. 1(21)/2012, pp. 163-176.

Zallony Marc-Philippe, (1824), Essai sur les Phanariotes, Marseille.

zanne Iuliu, (1901), Proverbele românilor, vol. 6, Bucureşti : Socec.

Zanne Iuliu, (2001), La Roumanie vue par les Français d'autrefois, București : Ed. Paula Romanescu, Éditions de la Fondation culturelle roumaine.

\section{NOTES}

1. Quelques exemples : « les Grecs sont une maladie tenace qui pénètre jusqu'aux os », "Avoir la tête vide comme un Grec ", "Grec galant homme, Juif idiot, Tsigane honnête, ça n'existe pas ", « vorace comme un Grec », « Le Grec allume son cigare au cierge de l'église », « le Grec langue de vipère ", "L'âne va dans les chardons et le Grec dans le jardin du voisin », " parler grec » au sens d'être ivre, etc. Cf. Iuliu Zanne, Proverbele românilor, vol. 6, 1901, p. 131-138.

2. Lucian Boia, Istorie şi mit în conştiinŢa românească, Humanitas, Bucureşti, 2011 (3éd.).

3. Nicolae Iorga, Byzance après Byzance [1935], Éditions Balland, Paris, 1992.

4. Mihai Eminescu, Opere, Publicistică, vol. v, Academia română, Univers enciclopedic, Bucureşti, 2000 , p. 744.

5. Idem, vol. IV, p. 116 (10 octobre 1876).

6. Nicolae Bălcescu, «Românii şi fanarioţii», Opere vol. I, Studii şi articole, Ed. Academiei Republicii Socialiste Române, București, 1953, p. 69-78.

7. Ibidem, p. 69.

8. Ibidem, p. 71. Marc-Philippe Zallony, Essai sur les Phanariotes, Marseille, 1824, p. 249. Précisons le sous-titre de cet ouvrage : «Où l'on voit les causes primitives de leur élévation aux hospodariats 
de Valachie et de Moldavie, leur mode d'administration et les causes principales de leur chute. » Sur l'influence de cet ouvrage, voir l'article de Jacques Bouchard dans le présent volume.

9. Nicolae Bălcescu, «Românii şi fanarioții», op. cit., p. 71.

10. Ibidem, p. 71.

11. Idem, "Question économique des principautés danubiennes », Opere, tomul I, partea a II-a, Scrieri istorice, politice şi economice, FundaŢia pentru literatură şi artă «Regelui Carol II», Bucureşti, 1940. p. 25.

12. Ibidem, p. 24

13. Nicolae Bălcescu, «Mersul revoluţiei...», Opere vol. I,. Studii şi articole, Ed. Academiei Republicii Populare Române, București, 1953, p. 307-313.

14. Ibidem, p. 308.

15. Ibidem, p. 308.

16. «ProclamaŢia revoluŢiunii din 9 iunie 1848 din Țara Românească », in Ion C. Brătianu, Acte şi cuvântări, vol. 1, op. cit., p. 473.

17. Voir par exemple Titu Maiorescu, «În contra direcţiei de azi în cultura română», Critice, Minerva, Bucureşti, 1984, p. 129.

18. Dumitru Drăghicescu, Din psihologia poporului român [1907], Albatros, Bucureşti, 1995, p. 258 (chap. 8, « L'Orient et son influence sur l'âme des Roumains »).

19. Ibidem, p. 269.

20. Lucian Boia, Istorie şi mit în conştiinţa românească, op. cit., p. 259-260.

21. Mihai Eminescu, III, p. 506.

22. Dumitru Drăghicescu, Din psihologia poporului român, op. cit., p. 241-257 (chap. 7 : « Les Grecs et les effets psychologiques de leur influence »).

23. Mihai Eminescu, vol. IV, op. cit., p. 115.

24. Ibidem, p. 705.

25. Ibid., p. 115.

26. Dumitru Drăghicescu, Din psihologia poporului român, op. cit., p. 211.

27. «Le peuple roumain n'a pas encore pensé, pour clouer le bec aux gouvernants de cette espèce, à instituer l'Ordre du saint Chanvre pour élever à la même dignité suspendue le Grec Serurie, le Grec C.A. Rosetti, le Bulgare Mihălescu et toute cette clique dominante. Mais ne désespérons pas. La plante pousse chez nous. » Eminescu, Opere vol. III, op. cit., p. 507-509.

28. Idem, vol. v, op. cit., p. 1142-1143.

29. Sur ce sujet, voir Gabriel Constantinescu, “The horrible crime' of Scarvulis (1880). Ethnical Stereotypes regarding the Greeks in Romania during the latter of the 19th Century" in Analele UniversităŢii din Craiova, Serie Istorie, Anul XVII, nr. 1(21)/2012, p. 163-176.

30. M. Eminescu, Opere, vol. v, op. cit., p. 1142. À propos de «croisements de peuple », on devine qu'il existe une situation dépassant toutes les autres en matière de "monstruosité » dans les fantasmes d'Eminescu : la rencontre entre Grecs et Juifs, ces deux vieux peuples passés par toutes les épreuves de l'histoire. Le résultat ne peut être qu'un cas monstrueux, dont Eminescu croit ici avoir trouvé un exemplaire dans la vie publique roumaine. Cela explique peut-être pourquoi on touche au comble de la violence verbale dans cet article qui est une attaque personnelle à l'égard d'un homme connu, article signé (ce qui est rare) et portant un titre (ce qui l'est également) : "Matériaux ethnologiques concernant en particulier M. Nicu Xenopulos, critique littéraire au Pseudo-Roumain». Il s'agit du frère de l'historien Alexandru Xenopol (ni grec ni Juif), critique littéraire au quotidien libéral Românul [le Roumain] dont le titre est typique, selon Eminescu, des stratégies de dissimulation et de tromperie des « étrangers roumanisés » qui le conduisent.

31. Dumitru Drăghicescu, Din psihologia poporului român, op. cit., p. 241-257.

32. Ibidem, p. 251.

33. Mihai Eminescu, Opere vol. III, op. cit., p. 514, p. 520, par exemple. 
34. Ibidem, p. 505.

35. Ibid., p. 513.

36. Idem, vol. v, op. cit., p. 864-865.

37. Id., vol. III, op. cit., p. 590.

38. Ibidem, p. 509.

39. Ibid., p. 731.

40. Idem, vol. v, op. cit., p. 751-752.

41. Id., vol. III, op. cit., p. 510 .

42. Ibid., p. 506.

43. Ibid., vol. III, op. cit., p. 525.

44. Ibid., p. 508.

45. Idem, vol. v, op. cit., p. 1253-1254.

\section{RÉSUMÉS}

À côté des Juifs, les Grecs représentent la minorité nationale la plus violemment visée par le discours des penseurs roumains de la seconde moitié du XIX ${ }^{\mathrm{e}}$ siècle. Il s'agit de préciser les contours, les fonctions et le sens de cette critique dans l'idéologie nationale de l'époque. Alors que Nicolae Bălcescu fait de l'épisode phanariote une phase de la marche dialectique de l'histoire roumaine, Mihai Eminescu et Dumitru Drăghicescu y voient dans leurs textes à dimension raciste la cause de tous les maux roumains, et certains conservateurs des années 1880 en font le schéma d'interprétation de la politique du parti libéral.

Alongside the Jews, the Greeks were the national minority that was the most violently targeted by Romanian thinkers in the latter half of the 19th century. This essay aims to outline the functions and significance of such a criticism within the framework of the national ideology of the time. If $\mathrm{N}$. Balcescu sees the phanariot episode as one of the stages in the dialectical progress of Romanian history, in their racially-oriented texts, M. Eminescu and D. Draghicescu see it as the root of all the evils Romania is confronted with. Finally, it also provided some conservativeminded thinkers with an interpretative prism they applied to the Liberal Party's policy in the 1880s. 
INDEX

Mots-clés : Phanariote, Bălcescu Nicolae (1819-1852), Grec, Phanariotes, Eminescu Mihai (1850-1889), Drăghicescu Dumitru (1875-1945)

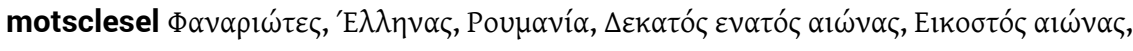

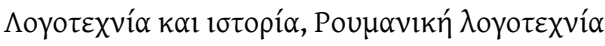
motsclestr Fenerliler, Yunan, Romanya, On dokuzuncu yüzyıllarda, Yirminci yüzyıllarda, Edebiyat ve Tarih, Romen edebiyatı

Keywords : Phanariot, Greek, Romania, Nineteenth century, Twentieth century, History and literature, Romanian literature

motsclesmk ФАНАР, ГРЧКА, РОМАНИЈА, ДЕВЕТНАЕСЕТТИОТ ВЕК, ДВАЕСЕТТИОТ ВЕК, ЛИТЕРАТУРА ВО ИСТОРИЈА, РОМАНСКИОТ ЛИТЕРАТУРА

\section{AUTEUR}

\section{CÉCILE FOLSCHWEILLER}

MCF

INALCO 